... Monika Hauser, Gynäkologin im Einsatz für kriegstraumatisierte Frauen, Trägerin des Alternativen Nobelpreises 2008

\title{
«lch habe meinen Beruf immer politisch verstanden»
}

Daniel Lüthi

Text und Bilder
Eine Frau, die viel unterwegs ist, ihrer Sache und sich selber aber stets treu: Das ist ein Eindruck, der sich bald einstellt und immer wieder bestätigt. Und dieser: eine Power-Frau - eine Person mit grosser Energie und eine, die dranbleibt. In der Schweiz geboren, kehrt sie ab und zu hierher zurück. Jetzt zum Beispiel für Vorträge in Luzern und Chur, für eine Vorstandssitzung der Schweizer Stiftung, die ihre Organisation «medica mondiale» [1] unterstützt - und für diese Begegnung am Sitz des Verlages, der die wohl wichtigste Publikation über sie herausgegeben hat. Der Titel des Buches ist Programm: «Nicht aufhören anzufangen»[2].

\section{Solidarität als Schlüssel}

Eine Reise nach Afrika steht bevor: In Liberia besucht Monika Hauser eines ihrer grösseren Projekte. In der Hauptstadt Monrovia will sie hochrangige Behördenmitglieder treffen, für ihre Sache Werbung machen, für ihre Anliegen Verständnis schaffen. Politische Arbeit und Lobbying - ja, das auch. Besonders wichtig aber ist ihr ein beschwerlicher Zweitages-Trip mit Jeep, der Besuch des Frauenzentrums in Fishtown, das auf ihre Initiative zurückgeht: «Ich brauche den direkten Kontakt zu unseren einheimischen Fachfrauen, will unsere Projekte regelmässig sehen, riechen, fühlen. Und: Immer wieder überbringe ich den Frauen

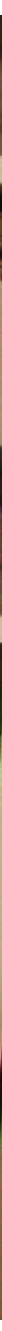


Grüsse von Kolleginnen, die anderswo Ähnliches erleben - das lässt sie Solidarität spüren.»

«Solidarität»: wohl DER Schlüsselbegriff in Monika Hausers Leben und Schaffen. «Die Solidarität ist meine Triebfeder. Sie gibt mir die Kraft, eine mühsame politische Arbeit leisten zu können.»

Vor ein paar Wochen weilte Hauser in Kabul, Afghanistan, wo sie unter anderem gegen die Zwangsverheiratung von Mädchen kämpft. Sie arbeitete in Bosnien und in Kosovo.

Aus der Gynäkologin, die in Innsbruck und Essen einst im Kreisssaal stand, ist eine international tätige und weltweit anerkannte Menschenrechts-Aktivistin geworden

Überall, immer wieder und auf den verschiedensten Ebenen geht es Monika Hauser ums Gleiche: um Mädchen und Frauen, die Gewalt erleiden, die vergewaltigt worden sind. Im Krieg, aber auch zu Hause. Darum, ihnen zuzuhören, ihnen zu helfen, sie zu begleiten und zu stärken. Und gleichzeitig in einer Gesellschaft, die oft nicht hinsehen und hinhören will, solche Gewalt anzuprangern und die Stimme der Opfer hörbar zu machen.

\section{Rebellin in einer Männerwelt}

Die erste Frau, die Monika Hauser von sexuellen Übergriffen berichtete, war ihre Grossmutter. Sie lebte in einem Dorf in Südtirol, Hauser war damals etwa 12-jährig. «Sie erzählte mir, wie Grossvater sie vergewaltigt hatte, um sie zu bekommen. Natürlich hat sie mir damit eine Last aufgebürdet. Aber sie hat mir auch die Augen geöffnet.» Auch für Übergriffe an sich selber: «Wie die meisten Mädchen erlebte auch ich Männer in der Verwandtschaft, die wie zufällig mit ihren Ellbogen meine Brüste streiften.» Hauser entwickelte Sensoren, um die Signale aufnehmen. Und die Bereitschaft, zu kämpfen. Als junge Assistenzärztin setzte sie sich dafür ein, dass der GynäkologieStuhl im Untersuchungszimmer gedreht, von der Türe abgewendet wurde, dass die Frauen, die mit gespreizten Beinen dalagen, nicht in unwürdiger Weise den direkten Blicken der Hereinkommenden ausgesetzt waren. «Schon damals eckte ich an mit meiner Kritik am patriarchalen System, das in der Medizin immer noch herrscht. Ich habe meinen Beruf immer politisch verstanden und ich war mit dem post-feudalistischen, technokratischen Gehabe in der Welt der Mediziner nie einverstanden.» Das tönt nach Revolution. «Nein, Revolution ist mir ein zu grosses Wort. Aber eine Rebellin - ja, das war ich immer.» Ein Arzt, eine Ärztin sei verpflichtet, ihren Patientinnen und Patienten zuzuhören, ist Monika Hauser überzeugt. «Und wir haben die gesellschaftliche Verpflichtung, das Wissen über Gewalt nach aussen zu tragen.»

Schon während ihrer klinischen Tätigkeit in den Achtzigerjahren habe sie erstaunt und verärgert festgestellt, wie wenig psycho-soziale und psycho-somatische Aspekte gerade in der Gynäkologie zählten,

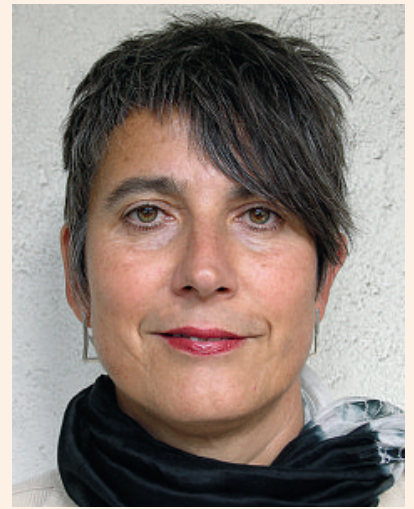

\section{Monika Hauser}

Dr. med. Monika Hauser wurde 1959 in St. Gallen geboren, wo sie auch aufwuchs, die Schulen besuchte und die Matura machte. In Innsbruck (Österreich) studierte sie Medizin, in Bologna (Italien) schloss sie ihr Studium mit dem Staatsexamen ab. In Essen (Deutschland) bildete sie sich dann zur Fachärztin für Gynäkologie weiter.

1992 erfuhr Monika Hauser via Medien von den Massenvergewaltigungen während des BalkanKrieges - Ende desselben Jahres reiste sie nach Bosnien, wo sie ein Therapiezentrum für vergewaltigte Frauen ins Leben rief und aufbaute. Anfang 1993 gründete sie die Frauenrechts- und Hilfsorganisation «medica mondiale». Als geschäftsführendes Vorstandsmitglied ist Monika Hauser dort heute verantwortlich für die fachlichen Inhalte sowie den Bereich Politik und Öffentlichkeitsarbeit. Ab 1999 arbeitete Hauser für kriegstraumatisierte Frauen in Kosovo und Albanien, es folgte der Aufbau neuer Projekte in Afghanistan und Liberia, den heutigen Schwerpunktländern von «medica mondiale».

2008 erhielt Monika Hauser den Alternativen Nobelpreis und damit weltweite Anerkennung.

Als Tochter von Südtiroler Eltern ist sie italienische Staatsbürgerin.

Wenn Monika Hauser nicht gerade unterwegs ist, lebt sie in Köln - zusammen mit Ehemann Klaus-Peter Klauner, der sich um den Haushalt kümmert und um den gemeinsamen 14-jährigen Sohn Luca. 
erzählt Monika Hauser in einem breiten Sankt Galler Dialekt oder Hochdeutsch mit Schweizer Akzent, wortgewandt und mit wachem Blick. «Wie oft wurden da bei unklaren Unterbauch-Beschwerden x Bauchhöhlenspiegelungen gemacht, die nichts ergaben. Dabei wäre es viel ergiebiger gewesen, die Patientinnen auf Gewalterfahrungen anzusprechen.»
Frauen reine Objekte.» Monika Hauser wollte Körper und Seelen heilen, wollte helfen - auch aufdecken helfen. Mit viel Elan, aber ohne etablierte Organisation im Rücken fuhr sie hin und wurde aktiv. Innerhalb weniger Monate schaffte sie es, das Frauentherapiezentrum Zenica aufzubauen - mit der Unterstützung lokaler Fachfrauen sowie deutscher

\section{«Die Macht liegt immer noch in den Händen von Männern, obschon eigentlich die Frauen das starke Geschlecht sind»}

Damals, vor 20 Jahren, waren es vor allem Männer, die in der Gynäkologie arbeiteten. «Bei den wenigen Ärztinnen gab es leider viele junge, intelligente Frauen, die sich wie Häschen verhielten. Sie biederten sich an, arbeiteten gratis, um weiterzukommen, sie ent-solidarisierten sich.» Inzwischen seien sowohl der Geist als auch die Quoten besser geworden, aber: «Die Medizin ist immer noch ein paternalistisches, post-feudales Gebiet. Es gibt inzwischen zum Glück zwar viele Gynäkologinnen, leider aber nach wie vor nur wenige Chefärztinnen. Die Macht liegt immer noch in den Händen von Männern, obschon eigentlich die Frauen das starke Geschlecht sind: Sie zeichnen sich aus durch kommunikative Fähigkeiten und Verhandlungsgeschick, durch Hartnäckigkeit, Durchhaltewillen und eine hohe Frustrationstoleranz.» Da spricht eine Frau, die gelernt hat, zu argumentieren und sich durchzusetzen. Sie tut es für tausende andere, die in ihrer Lebenslage keine Kraft haben, sich für sich selbst einzusetzen.

Wie von einer anderen Welt sind da Luxus-Elemente der modernen Gynäkologie, In-vitro-Fertilisation oder Intim-Chirurgie zum Beispiel: «Labien oder Brüste $\mathrm{zu}$ vergrössern und $\mathrm{zu}$ verkleinern - damit habe ich grösste Mühe, wenn es medizinisch nicht indiziert ist. Wir müssen den Frauen Mut machen, sich nicht über ihren Körper zu definieren.»

\section{Körper und Seelen heilen}

Das heutige kompromisslose Engagement von Monika Hauser begann 1993, mit den Greueln der BalkanKriege, den Massen-Vergewaltigungen in Bosnien. «In den Medien sah ich Blut, Tränen, Schande. Mit dem Schlüsselloch-Blick wurde nur auf die Vergewaltigungen geschaut, wieder einmal waren die
Kolleginnen und von ihrem späteren Ehemann, dank öffentlichen Geldern und privaten Spenden. Schwer traumatisierte Frauen wurden medizinisch und psychologisch betreut, Schutz- und Wohnhäuser wurden ihnen zur Verfügung gestellt. Die bosnische Organisation «Medica Zenica» entstand daraus, kurz darauf gründete Hauser in Deutschland «medica mondiale».

«Die Projekte in Bosnien, Kosovo und Albanien laufen inzwischen weitgehend unabhängig», freut sich Monika Hauser. Natürlich freut sie sich auch über den Alternativen Nobelpreis, den sie 2008 erhalten hat, er hat ihr und ihren Anliegen zu grosser Publizität verholfen. «Speziell bei Männern, bei Politikern, die nicht bereit sind, ihre Verantwortung wahrzunehmen, hilft uns diese Auszeichnung, uns Gehör zu verschaffen.»

Auch einer starken Frau wie Monika Hauser kann bisweilen alles zu viel werden. «1995 hatte ich einen schweren Zusammenbruch, da ging bei mir physisch und psychisch nichts mehr.» Was ihr auch damals besonders zu schaffen machte: Ignoranz und Gleichgültigkeit. Auch sich selber gegenüber wollte Hauser nicht gleichgültig sein. «Ich merkte, dass das Gift der Gewalt auch in mir wirkt. Und dass es eine stellvertretende Traumatisierung zu vermeiden gilt.» Gemeinsam mit Kolleginnen entwickelte sie ein Massnahmen-Paket zum Selbstschutz: «Eine regelmässige professionelle Supervision ist für mich selbstverständlich geworden. Ich pflege bewusst meine Hobbies, gehe joggen und spiele Saxophon. Und: Ich verbünde mich mit Gleichgesinnten.»

Womit wir wieder beim Schlüsselbegriff in Monika Hausers Leben und Schaffen sind: Solidarität.
1 www.medicamondiale.org

2 Louis C. Monika Hauser Nicht aufhören anzufangen. Zürich: Verlag rüffer \& rub; 2008
Die nächste «Begegnung mit ...»

Am Ende jeden Monats stellt die Schweizerische Ärztezeitung eine Persönlichkeit vor, die sich im Gesundheitswesen engagiert. Anlässlich des Welt-Aids-Tages schildert Daniel Lüthi im November seine Begegnung mit Ruedy Lüthy, Infektiologe, Aids-Spezialist der ersten Stunde, Klinik-Leiter in Simbabwe und Ehrendoktor der Universität Bern. 